\title{
A Hessian free method to prevent zero-point energy leakage in classical trajectory simulation
}

\author{
Saikat Mukherjee ${ }^{a, 1^{*}}$, Mario Barbatti ${ }^{a, b, 2^{*}}$ \\ ${ }^{a}$ Aix Marseille University, CNRS, ICR, Marseille, France \\ ${ }^{b}$ Institut Universitaire de France, 75231 Paris, France \\ ${ }^{1}$ ORCID: 0000-0002-0025-4735 \\ ${ }^{2}$ ORCID: 0000-0001-9336-6607 \\ *saikat.mukherjee@univ-amu.fr, mario.barbatti@univ-amu.fr
}

Keywords: zero-point energy, prevent zero-point energy leak, Hessian free method, classical trajectory simulation

\begin{abstract}
The problem associated with the zero-point energy (ZPE) leak in classical trajectory calculations is well known. Since ZPE is a manifestation of the quantum uncertainty principle, there are no restrictions on energy during the classical propagation of nuclei. This phenomenon can lead to unphysical results, such as forming products without the ZPE in the internal vibrational degrees of freedom (DOFs). The ZPE leakage also permits reactions below the quantum threshold for the reaction. We have developed a new Hessian-free method, inspired by the Lowe-Andersen thermostat model, to prevent energy dipping below a threshold in the local-pair (LP) vibrational DOFs. The idea is to pump the leaked energy to the corresponding local vibrational mode, taken from the other vibrational DOFs. We have applied the new correction protocol on the ab initio ground-state molecular dynamics simulation of the water dimer $\left(\mathrm{H}_{2} \mathrm{O}\right)_{2}$, which dissociates due to unphysical ZPE spilling from the high-frequency OH modes. The LP-ZPE method has been able to prevent the ZPE spilling of the $\mathrm{OH}$ stretching modes by pumping back the leaked energy into the corresponding modes while this energy is taken from the other modes of the dimer itself, keeping the system as a microcanonical ensemble.
\end{abstract}




\section{Introduction}

Zero-point energy (ZPE) is a consequence of the quantum uncertainty principle, and thereby, an inherent error creeps into the propagation of classical trajectories in mixed quantum-classical (MQC) methods by neglecting it. The classical nuclear motion approximation permits reactions below the quantum threshold of energy leading to unphysical results where products can be formed with less energy than ZPE in the internal vibration degrees of freedom. The problem becomes serious if the ZPE flows out of several modes and pumps into a specific weak bond to make it unphysically hot. This ZPE leakage phenomenon is well discussed in the literature, ${ }^{1-6}$ and few strategies have been designed to reinforce ZPE maintenance during quasi-classical trajectories (QCT).

Independently, Bowman et al. $^{7}$ and Miller \& Hase ${ }^{8,9}$ proposed a method to constrain the ZPE spilling by time reversal of the momentum of any normal mode undergoing ZPE leakage. In other words, they imposed a hard-wall sphere in the phase space, inside of which the energy is less than the ZPE of the corresponding normal mode. Whenever a mode loses energy and the resultant trajectory tends to enter the hard-wall sphere, it is forced to bounce back. This protocol can be done by monitoring the normal harmonic modes at each timestep and reversing the sign of the momentum of the particular mode, which drops its energy below the corresponding ZPE. However, this treatment is valid only when the system is not far from the well-defined equilibrium region. The same algorithm should be applied at any arbitrary nuclear configuration outside the equilibrium region on the instantaneous normal modes. However, the method has some apparent shortcomings as the impulsive kicks produce discontinuous linear momenta and vibrational phase of the altered mode.

Lim et al. modified ${ }^{10}$ the hard-sphere model by a rigid but inelastic wall sphere, where the trajectory is repelled from entering the quantum forbidden zone and is made to "skid" along the ZPE orbit. This prescription, namely, trajectory projection onto ZPE orbit (TRAPZ), was achieved by applying a non-Newtonian force in holonomic constraints dynamics, which showed that each corrected mode's momentum and vibrational phase are continuous. TRAPZ method was used to study the dissociation of the $\mathrm{Al}_{3}$ cluster and found a highly vibrationally excited molecular fragment of $\mathrm{Al}_{2}{ }^{11}$. The overestimation can be rationalized because each mode has to conserve its ZPE even if all the other modes have energy much higher than their ZPE. mTRAPZ ${ }^{12}$ is a softened version of TRAPZ, where the linear momenta transformation is 
only performed when the total instantaneous vibrational energy drops below the total local ZPE. In other words, now the checking of ZPE leaking is carried out not for individual modes but for the total local vibrational energy of the system to prevent excess adding of vibrational energy at the expense of translational energy.

Varandas modified the Bowman-Hase-Miller approach of constraining ZPE in quasiclassical trajectory calculations by compensating the amount of energy leak of a bound vibrational mode from the remaining modes of the molecular system. ${ }^{13}$ The protocol is the following: At each time step, the local normal mode coordinates (instantaneous normal modes) are determined, and the amount of energy leak for each vibrational mode is calculated. This leaked energy will be given to the local normal mode, withdrawn from the energy pool constructed by the sum of energy in the remaining vibrational modes.

Keeping in mind that the intermode coupling terms in a multimode Hamiltonian govern the energy transfer between the modes, Xie et al. proposed ${ }^{14}$ a method of annihilating mode coupling by introducing a smooth switching function when the energy of the corresponding mode drops below the ZPE. In principle, this method can be applied to the onthe-fly dynamics of a general polyatomic system. However, it has not yet been tested in this context.

In the past decade, the quantum thermal bath (QTB) model ${ }^{15}$ was introduced to quantify the ZPE leakage in molecular simulations using colored-noise Langevin dynamics. In this framework, temporal prevention of the ZPE leakage could also be achieved by increasing the damping coefficient value, but the simulation's accuracy will be questionable. Recently, an adaptive QTB model (adQTB) has been proposed ${ }^{16,17}$ to suppress the ZPE leakage phenomenon, where quantum fluctuation-dissipation criterion is actively enforced by adjusting the system-bath coupling parameter all along the simulation. In other words, instead of a constant coupling, an "on-the-fly" tuned frequency-dependent coupling parameter is used to construct the Langevin equation of motion. As a result, each vibrational mode of the system is thermalized at an effective temperature that includes the appropriate ZPE, and thereby, the dynamics always accounts for the quantum ZPE effect.

A different approach ${ }^{18}$ was taken to conserve the total ZPE throughout a trajectory by constructing an effective potential by adding an estimate of harmonic ZPE at an arbitrary nuclear configuration to the Born-Oppenheimer (BO) molecular potential energy surface (PES). Thus, an effective ZPE-corrected PES was constructed for the $\mathrm{H}_{2} \mathrm{CO}$ molecule, and 
QCT simulations on this modified PES yielded good product state distributions for the roaming reaction.

Apart from the above discussed "active" ZPE constraint models, few "passive" techniques have been suggested to overcome the ZPE leaking problem in classical trajectory calculations. ${ }^{19-22}$ These methods do not alter the dynamics but discard unphysical trajectories from the statistics that either form products with less than their ZPE or form products where the sum of the product vibrational energies is less than the total product ZPE.

All "active" ZPE constraint models discussed above (except adQTB) are based on calculating instantaneous normal modes and Hessians. Because the dynamics of realistic molecules is usually based on on-the-fly calculation of electronic quantities and potential energy surfaces are not pre-computed, to require knowing the Hessian during the propagation becomes an insurmountable hurdle even for medium sized-molecules. Moreover, we miss a method that could also be adapted to mixed-quantum classical methodologies based on BornOppenheimer molecular dynamics (BOMD), like surface hopping. ${ }^{23}$ Thus, the conclusion of Guo et al. ${ }^{5}$ that no satisfactory solution to the infamous ZPE spilling problem in classical dynamics has been found to date remains somewhat true 25 years later. Novel techniques devoided of Hessian calculations, tailored to on-the-fly dynamics, and generally adaptable to different dynamics algorithms must be developed if we aim for long timescale simulation. ${ }^{24}$

In this article, we take the first steps to close this methodological gap. We present a new Hessian free method for correcting the ZPE leakage in a molecule during classical or MQC dynamics in Section 2. Named Local-Pair (LP) ZPE correction, it is applied on the water dimer $\left(\mathrm{H}_{2} \mathrm{O}\right)_{2}$ system, where the dimer dissociates due to unphysical ZPE spilling from the high-frequency $\mathrm{OH}$ modes. The computational details are given in Section 3. We discuss the results obtained from unconstrained and constrained trajectory simulations in Section 4. Finally, we conclude about the outlook of the method in Section 5.

\section{Theory}

The LP-ZPE correction method is a collisional model inspired by the Lowe-Andersen thermostat. ${ }^{25}$ It monitors the local vibration of hydride modes $(\mathrm{AH})$, where $\mathrm{A}$ is any atom without losing any generality. When the kinetic energy of such vibration drops below the zero-point energy of the $\mathrm{AH}$ mode, the kinetic energy difference is added to the AH bond. To compensate, the excess energy is removed from an energy pool constructed by the other atoms of the molecule. In contrast with the thermostat model, the energy is taken from the 
molecule itself, and the velocity change conserves the total energy, keeping the system as a microcanonical ensemble. The workflow can be described as follows:

(1) The atom pairs of the molecule are classified into two groups: (a) The high frequency modes built from the atom pairs, mainly prone to ZPE leakage (such as hydride bonds), are named as $\mathrm{AH}$ bonds. These $\mathrm{AH}$ bonds will be monitored for ZPE leakage, and if necessary, energy will be added to them. (b) All the other atom pairs, not necessarily bonded, are named BC pairs. These atom pairs will donate energy to the energy pool from where energy will be pumped to the leaked bond.

(2) The average parallel component of the $\mathrm{A}$ and $\mathrm{H}$ velocities to the kinetic energy for each $\mathrm{AH}$ bond is calculated at the beginning of the dynamics over time $\tau$.

$$
\bar{\varepsilon}_{k}^{0}=\frac{1}{N} \sum_{t=0}^{\tau} \frac{1}{2} \mu_{A H}\left[v_{H}^{\|}(t)-v_{A}^{\|}(t)\right]^{2}
$$

where $N$ is the number of steps. $\mu_{A H}$ is the reduced mass of $\mathrm{AH}$ bond defined as $\mu_{A H}=m_{A} m_{H} /\left(m_{A}+m_{H}\right)$ with $m_{A(H)}$ being the mass of the $\mathrm{A}(\mathrm{H})$ atom. The parallel component of the velocity of A atom is $v_{A}^{\|}=\mathbf{v}_{A} \cdot \hat{\mathbf{u}}_{A H}$, and $\hat{\mathbf{u}}_{A H}=\mathbf{R}_{A}-\mathbf{R}_{H} /\left\|\mathbf{R}_{A}-\mathbf{R}_{H}\right\|$. We use bold letters for vectors and matrices throughout the manuscript.

(3) At later time $t_{c}$, the mean contribution of the parallel velocity components to the kinetic energy for each $\mathrm{AH}$ bond is again calculated over the same time period $\tau$,

$$
\bar{\varepsilon}_{k}^{c}=\frac{1}{N} \sum_{t=t_{c}}^{t_{c}+\tau} \frac{1}{2} \mu_{A H}\left[v_{H}^{\|}(t)-v_{A}^{\|}(t)\right]^{2}
$$

(4) If the mean kinetic energy drops below a pre-defined critical threshold value $\varepsilon$,

$$
\delta \varepsilon=\bar{\varepsilon}_{k}^{0}-\bar{\varepsilon}_{k}^{c}>\varepsilon
$$

the leaked energy is added to the $\mathrm{AH}$ bond in terms of the velocities of $\mathrm{A}$ and $\mathrm{H}$ atoms as

$$
\begin{aligned}
& \mathbf{v}_{H_{\text {new }}}=\mathbf{v}_{H_{\text {old }}}+\frac{\mu_{A H}}{m_{H}} \Delta_{A H} \hat{\mathbf{u}}_{A H}, \\
& \mathbf{v}_{A_{\text {new }}}=\mathbf{v}_{A_{\text {old }}}-\frac{\mu_{A H}}{m_{A}} \Delta_{A H} \hat{\mathbf{u}}_{A H},
\end{aligned}
$$

where 


$$
\Delta_{A H}=\left[\left(v_{H_{\text {old }}}^{\|}-v_{A_{\text {old }}}^{\|}\right)^{2}+\left(\frac{2 \delta \varepsilon}{\mu_{A H}}\right)\right]^{1 / 2}-\left(v_{H_{\text {old }}}^{\|}-v_{A_{\text {old }}}^{\|}\right) .
$$

(5) The same amount of pumped energy is removed from each of the BC atom pairs (not containing an $\mathrm{H}$ atom) by adjusting the velocities of $\mathrm{B}$ and $\mathrm{C}$ atoms as

$$
\begin{aligned}
& \mathbf{v}_{B_{\text {new }}}=\mathbf{v}_{B_{\text {old }}}+\frac{\mu_{B C}}{m_{B}} \Delta_{B C} \hat{\mathbf{u}}_{B C}, \\
& \mathbf{v}_{C_{\text {new }}}=\mathbf{v}_{C_{\text {old }}}-\frac{\mu_{B C}}{m_{C}} \Delta_{B C} \hat{\mathbf{u}}_{B C},
\end{aligned}
$$

where

$$
\Delta_{B C}=\left[\left(v_{B_{\text {old }}}^{\|}-v_{C_{\text {old }}}^{\|}\right)^{2}-\left(\frac{2 \delta \varepsilon}{\mu_{A H}} f_{B C}\right)\right]^{1 / 2}-\left(v_{B_{\text {old }}}^{\|}-v_{C_{\text {old }}}^{\|}\right) .
$$

The term $f_{B C}$ is a weight factor, which determines how much energy is taken from the corresponding $\mathrm{BC}$ atom pair among all the $B^{\prime} C^{\prime}$ atom pairs to construct the energy pool. There is some methodological freedom to choose this function. In this first application of the LP-ZPE method, it is defined as

$$
f_{B C}=\frac{\frac{1}{2} \mu_{B C}\left(v_{B_{\text {old }}}^{\|}-v_{C_{\text {old }}}^{\|}\right)^{2}}{\sum_{B^{\prime} C^{\prime}} \frac{1}{2} \mu_{B^{\prime} C^{\prime}}\left(v_{B_{\text {old }}^{\prime}}^{\|}-v_{C_{\text {old }}^{\prime}}^{\|}\right)^{2}} .
$$

(6) A problematic situation may arise when there is no sufficient kinetic energy available in a $\mathrm{BC}$ atom pair, i.e.

$$
\left(v_{B_{\text {old }}}^{\|}-v_{C_{\text {old }}}^{\|}\right)^{2}-\left(\frac{2 \delta \varepsilon}{\mu_{A H}} f_{B C}\right)<0,
$$

then, the velocities of that particular BC pair are not changed. In that case, the weight factor $f_{B C}$ is recomputed, excluding the $\mathrm{BC}$ pair with imaginary component and the same amount of pumped energy $(\delta \varepsilon)$ is removed from the other BC pairs to maintain the energy conservation.

$$
\varepsilon_{\text {removed }}=\sum_{B^{\prime} C^{\prime}}\left(\delta \varepsilon f_{B^{\prime} C^{\prime}}\right)
$$

where the sum excludes the BC pair with imaginary components. 
Following this prescription, the LP-ZPE correction poses a minimum impact on the dynamics, as we will discuss. The total energy, total linear momentum, and the angular momentum of the center of mass are perfectly conserved, as we show in the Appendices.

The main advantage of the LP-ZPE correction over most other approaches is that it does not require the computation of Hessian matrices because the ZPE leakage is monitored for local AH pairs, not normal modes. We can justify using local AH stretching vibrations as the basis for applying ZPE corrections by noticing that the high-frequency normal modes can be well described in terms of local fragment modes. ${ }^{26}$ For instance, Huix-Rottlant and Ferré ${ }^{26}$ showed that the high-frequency normal modes of benzene are combinations of single internal local oscillators (the local oscillator matrix appears as a Huckel-like structure), where each local stretching oscillator couples only to the nearest neighbor with a fixed coupling value. Thus, the eigenvectors of such a local oscillator matrix lead to a spectrum that closely matches the six highest normal modes of benzene, obtained by the full Hessian-matrix diagonalization.

\section{Computational Details}

As reviewed by Bowman et al., the absence of ZPE in classical MD simulations involving water can be severely erroneous. ${ }^{27}$ With this motivation, we applied our LP-ZPE correction method to prevent the ZPE leakage in BOMD of the water dimer system. We have employed MP2/aug-cc-pVDZ level of theory in this work for ground-state optimization of the dimer, normal mode analysis at the optimized ground state geometry, and ground-state BOMD. The dimer dissociation energy obtained in this work is $0.225 \mathrm{eV}$ (Table 1), which is much larger than the ZPE $(0.012 \mathrm{eV})$ of the stretching mode (the harmonic frequency is 189 $\mathrm{cm}^{-1}$; see Table S1), causing dissociation of the water dimer. So, any dissociation of the dimer during the classical dynamics should occur only due to the unrealistic ZPE leakage from the high-frequency $\mathrm{OH}$ stretching modes. 
Table 1. Geometric properties (Angstrom, degree) and dissociation energy (eV) of water dimer in this present calculation are compared with previously reported results.

\begin{tabular}{|l|l|l|l|l|}
\hline & \multirow{2}{*}{$\begin{array}{l}\text { Present result } \\
\text { MP2/aug-cc-pVDZ }\end{array}$} & \multicolumn{2}{|l|}{ Previous theoretical results } & \multirow{2}{*}{ Previous expt. results $^{28}$} \\
\cline { 3 - 4 } & & $\begin{array}{l}\text { a CCSD(T)- } \\
\text { F12b/CBS }\end{array}$ & $\begin{array}{l}\text { b CCSD(T)(FU } \\
\text { LL)/IO275 }\end{array}$ & \\
\hline $\mathrm{R}\left(\mathrm{O}_{1} \mathrm{O}_{4}\right)$ & 2.90758 & 2.91278 & 2.912 & $2.976(+0.0000,-0.030)$ \\
\hline $\mathrm{R}\left(\mathrm{O}_{1} \mathrm{H}_{2}\right)$ & 0.96617 & 0.95902 & 0.9583 & \\
\hline $\mathrm{R}\left(\mathrm{O}_{4} \mathrm{H}_{5}\right)$ & 0.97249 & 0.96464 & 0.9639 & \\
\hline $\mathrm{R}\left(\mathrm{O}_{4} \mathrm{H}_{6}\right)$ & 0.96427 & 0.95744 & 0.9569 & \\
\hline$\theta\left(\mathrm{H}_{2} \mathrm{O}_{1} \mathrm{H}_{3}\right)$ & 104.136 & 104.892 & 104.87 & \\
\hline$\theta\left(\mathrm{H}_{5} \mathrm{O}_{4} \mathrm{H}_{6}\right)$ & 104.450 & 104.801 & 104.83 & \\
\hline$\alpha$ & 7.402 & 5.703 & 5.5 & $6 \pm 20$ \\
\hline$\beta$ & 113.506 & 123.379 & 124.4 & $123 \pm 10$ \\
\hline $\begin{array}{l}{ }^{c} \text { Dissociation } \\
\text { energy }\left(D_{e}\right)\end{array}$ & 0.225 & 0.216 & 0.2177 & ${ }^{\mathrm{d}} 0.236 \pm 0.03$ \\
\hline
\end{tabular}

${ }^{\mathrm{a}} \mathrm{CCSD}(\mathrm{T})-\mathrm{F} 12 \mathrm{~b} / \mathrm{CBS}$ extrapolation scheme with the aug-cc-pVQZ/aug-cc-pV5Z basis sets.

${ }^{\text {b }} \mathrm{CCSD}(\mathrm{T})(\mathrm{FULL}) / \mathrm{IO} 275$ (IO275: interaction optimized basis set with 275 basis functions for the $\mathrm{H}_{2} \mathrm{O}$ dimer. O:

7s5p5d3f2g1h; $\mathrm{H}_{\mathrm{d}}$ : 2s4p1d, H: 2s3p, BF: 3s3p2d1f).

${ }^{\mathrm{c}} D_{e}$ is the total bond energy (BSSE corrected) from the bottom of the well.

${ }^{\mathrm{d}}$ Reference ${ }^{31}$. $D_{e}$ was estimated by adding the ZPE calculated at the HF/4-21G level.

We have sampled 100 initial conditions from correlated quantum-harmonic oscillator distribution such that the total energy of the system lies within the energy window of ZPE \pm $0.1 \mathrm{eV}$ (see details in the Supporting Information (SI)). Figure S1 depicts the distribution of the total energy of the selected 100 initial conditions out of a total of 500 sampled points. Ground-state classical trajectory simulations are carried out with the 100 initial conditions by a homegrown newly written Newton-X suit of $\operatorname{codes}^{32,33}$ (Newton-X NS) in two ways: (a) without any constrain or ZPE leakage correction and (b) applying the LP-ZPE correction in the dynamics. In both cases, classical trajectories are propagated via Newtonian equations of motion up to $20 \mathrm{ps}$ with the velocity-Verlet algorithm with a timestep of 0.5 fs. The forces acting on the nuclei are proportional to the gradient of the ground state BO potential energy surface, where the latter is calculated on the fly by MP2/aug-cc-pVDZ theory using Turbomole (version 7.3) suite of quantum chemistry programs. ${ }^{34,35}$

In the LP-ZPE correction scheme on water dimer, we identified the four $\mathrm{OH}$ bonds as the local AH pairs, and all the other 11 atom pairs (even not bonded) are considered BC atom 
pairs. Hence, the four local AH pairs are $\left(\mathrm{O}_{1}-\mathrm{H}_{2}\right),\left(\mathrm{O}_{1}-\mathrm{H}_{3}\right),\left(\mathrm{O}_{4}-\mathrm{H}_{5}\right),\left(\mathrm{O}_{4}-\mathrm{H}_{6}\right)$, and the eleven BC atom pairs are $\left(\mathrm{O}_{1}-\mathrm{O}_{4}\right),\left(\mathrm{O}_{1}-\mathrm{H}_{5}\right),\left(\mathrm{O}_{1}-\mathrm{H}_{6}\right),\left(\mathrm{H}_{2}-\mathrm{H}_{3}\right),\left(\mathrm{H}_{2}-\mathrm{O}_{4}\right),\left(\mathrm{H}_{2}-\mathrm{H}_{5}\right),\left(\mathrm{H}_{2}-\mathrm{H}_{6}\right),\left(\mathrm{H}_{3}-\mathrm{O}_{4}\right)$, $\left(\mathrm{H}_{3}-\mathrm{H}_{5}\right),\left(\mathrm{H}_{3}-\mathrm{H}_{6}\right),\left(\mathrm{H}_{5}-\mathrm{H}_{6}\right)$ as shown in Figure 1. Since non-bonded atom pairs are considered in the BC pair ensemble, energy is pumped from all types of vibrations (and not only stretching modes) into the leaked modes.

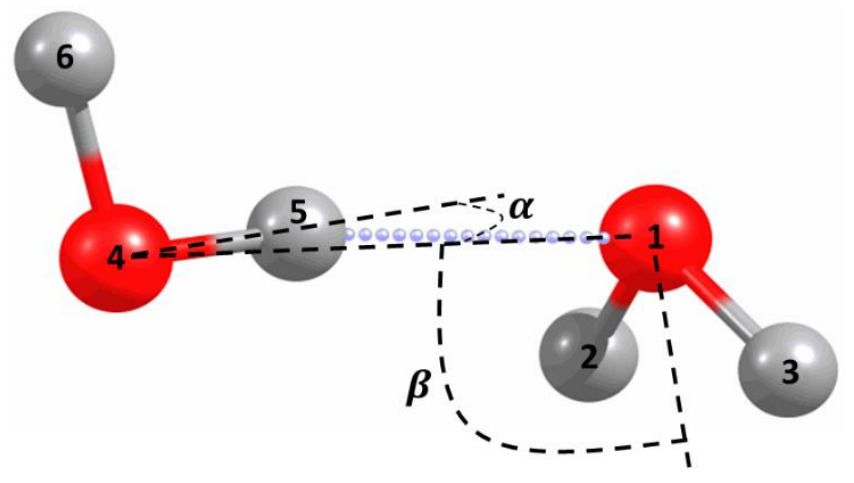

Figure 1. The equilibrium structure of the water dimer.

We have chosen the three parameters needed to set up this model as (a) time period for averaging the parallel component of the kinetic energy of AH bond, $\tau=10 \mathrm{fs}$, (b) At each $t_{c}=10 \mathrm{fs}$, checking of ZPE leakage is invoked, and (c) $\varepsilon=0.001 \mathrm{a}$.u. is taken as the maximum allowed deviation of the mean kinetic energy at the correction time from the reference value obtained at the beginning of the dynamics (Eq. (2)).

A judicial and careful parameter setting is needed to apply LP-ZPE correction scheme. The time period for averaging the parallel component of the kinetic energy of AH bond $(\tau)$ should allow the $\mathrm{OH}$ stretching to complete a few oscillations, and the energy can deliver a reasonable estimate of the ZPE value of the local vibration mode. It can be decently assumed that there should not be much ZPE leakage at the beginning of the dynamics. The parameter $t_{c}$-Also controlling the correction time during the dynamics - should not be too short. Otherwise, unnecessary corrections could hamper the inherent dynamics. On the other hand, if $t_{c}$ is too long, it would lose the essence of the correction. The last parameter, $\varepsilon$, a pre-defined threshold value, defines how much kinetic energy loss permissible in the local AH bonds from the corresponding reference ZPE value. Again, a very low threshold would force the method to interfere much with the inherent dynamics, and a high value does not allow the correction to occur. This work allowed $\sim 10 \%$ energy loss from the corresponding ZPE value of the highfrequency AH bonds. Although more tests concerning the parameters will be carried out in the 
future, we expect a moderate set of parameters chosen in this work is adequate to demonstrate the workability of the LP-ZPE corrections in classical trajectory calculation.

To validate our ground state classical dynamics, we have simulated the power spectra from the classical trajectories by Fourier transform the velocity autocorrelation function using TRAVIS software, ${ }^{36,37}$

$$
P(\omega)=m \int\langle\dot{\mathbf{r}}(\tau) \dot{\mathbf{r}}(t+\tau)\rangle_{\tau} e^{-i \omega t} d t,
$$

where $m$ is the reduced mass of the oscillator and $\dot{\mathbf{r}}$ is the velocity vector.

We have codified the LP-ZPE correction scheme in a Fortran 2008 written module, which can be interfaced readily with any classical trajectory propagator. The LP-ZPE correction code mainly requires the nuclear geometries to determine the $\mathrm{AH}$ bond and $\mathrm{BC}$ atom pairs, and velocities to add and remove energies from $\mathrm{AH}$ bond and $\mathrm{BC}$ atom pairs, respectively, at the correction timestep. The code is distributed freely with this article.

\section{Results and Discussion}

Water dimer, a prototypical hydrogen-bonded complex, has been serving as a classic testbed for benchmarking the electronic structure methods for more than twenty years, not only because of its small size but also its fundamental physical properties and important role in atmospheric chemistry. The interested readers are referred to the detailed chronological reviews of experimental ${ }^{38}$ and theoretical ${ }^{39}$ studies on water dimer by Mukhopadhyay et al. Since a detailed discussion ${ }^{40-42}$ on the electronic structure calculation of water dimer is outside the scope of this article, we limit ourselves by comparing only the geometry, harmonic normal mode frequencies, and the dissociation energy of our optimized cluster with previously reported theoretical and experimental findings (Table 1 and Table S1). As expected, compared to the sophisticated electronic structure methods with high basis sets, our results obtained by MP2/aug-cc-pVDZ level of theory are not the best estimates ${ }^{42}$ but fall within the margin of errors. Hence, we can safely proceed with the present level of theory and apply our LP-ZPE correction during the propagation of the classical trajectories.

Figure 2 shows the distance between the oxygen atoms of the monomers as a function of time for the 100 classical trajectories in grey lines for both types of dynamics, with and without the LP-ZPE correction. The monomeric distances are averaged over the 100 trajectories in both cases and shown in solid black lines. It is evident that if we do not prevent the ZPE leakage from the $\mathrm{AH}$ bonds (in this case, the fast $\mathrm{OH}$ stretching modes), the dimer 
starts to dissociate rapidly after 4 ps. The same phenomenon was reported ${ }^{27}$ previously by Bowman and his group. Applying our LP-ZPE correction scheme, most of the trajectories do not dissociate up to the end of the simulation, 20 ps. It can be argued that those few trajectories still showing large distances between the monomers even after applying the LPZPE correction, undergoing energy leakage occurred from modes other than stretching, such as bending, wagging, shearing type of motions.
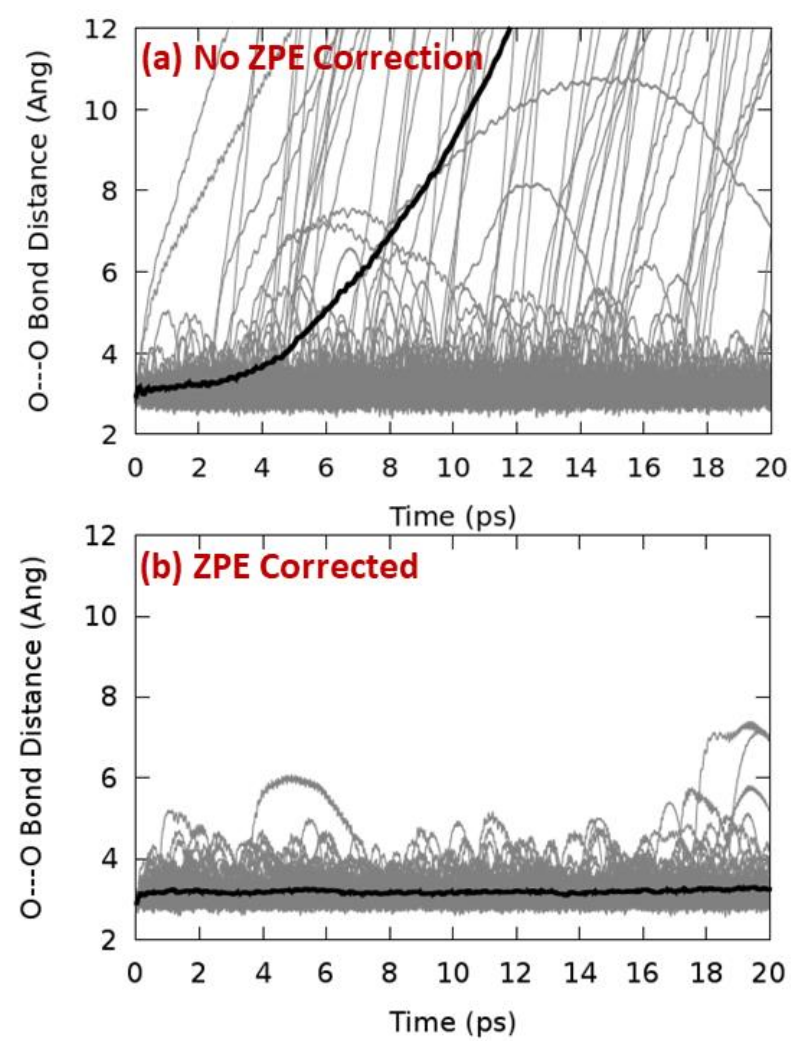

Figure 2. The distances between the two oxygen atoms of the monomers as a function of time (grey lines) for the trajectories without ZPE correction are shown in panel (a). The same quantity is shown in panel (b) for trajectories starting from the same initial conditions but with LP-ZPE correction. The thick back lines represent the averaged distances between the two monomers $\left(\mathrm{O}_{1}-\mathrm{O}_{4}\right)$ in both cases.

The radial distribution functions (RDFs) of the two oxygen atoms in Figure 3 also show that dissociation of the dimer prevails in the trajectories calculated without applying any ZPE correction. Since the O-O distance of water dimer in equilibrium is 2.91 Angstrom, the RDFs peak around 3 Angstrom in both the cases. The RDF obtained from the trajectories without ZPE correction (blue curve) leaves a long tail in higher $\mathrm{O}-\mathrm{O}$ distances implying 
dissociation of the trajectories, whereas the RDF for ZPE corrected trajectories ends within 6 Angstrom.

Figure 4 shows the averaged power spectrum over 100 trajectories with the LP-ZPE correction scheme. The frequencies obtained from the power spectra calculated from groundstate BOMD trajectories lie within the acceptable range of the scaled ${ }^{43}$ normal mode frequencies of water dimer molecule calculated in this work, thereby validating our LP-ZPE correction protocol on classical trajectory simulation. A peak around $3200 \mathrm{~cm}^{-1}$ is seen in the power spectrum, which does not correspond to any normal mode frequencies of the water dimer. It approximately matches the frequency of the hydroxyl cation $\left(\mathrm{OH}^{+}\right)$, whose experimental value in solid Argon matrix is $2979.6 \mathrm{~cm}^{-1}$, and the scaled value calculated by MP2/6-31++G(d,p) level of theory is $3110 \mathrm{~cm}^{-1} .{ }^{44}$ This peak may indicate some residual artifact from the LP-ZPE correction.

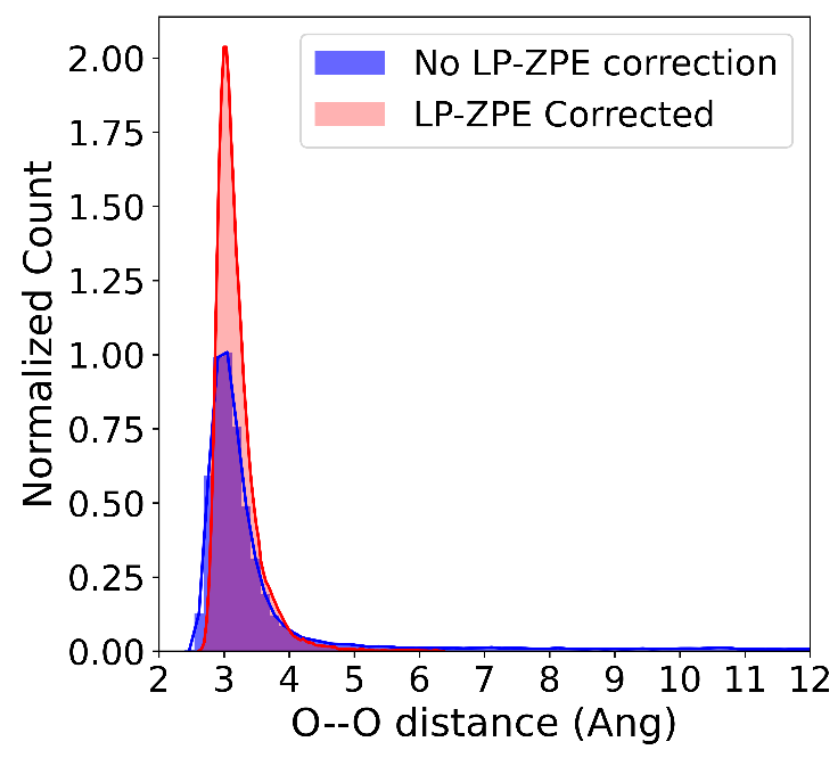

Figure 3. The radial distribution functions (RDFs) of $\mathrm{O} 1-\mathrm{O} 4$ atoms in water dimer calculated from trajectories with (red) and without (blue) the LP-ZPE correction. Each RDF is normalized such that the area under each curve is unity. 


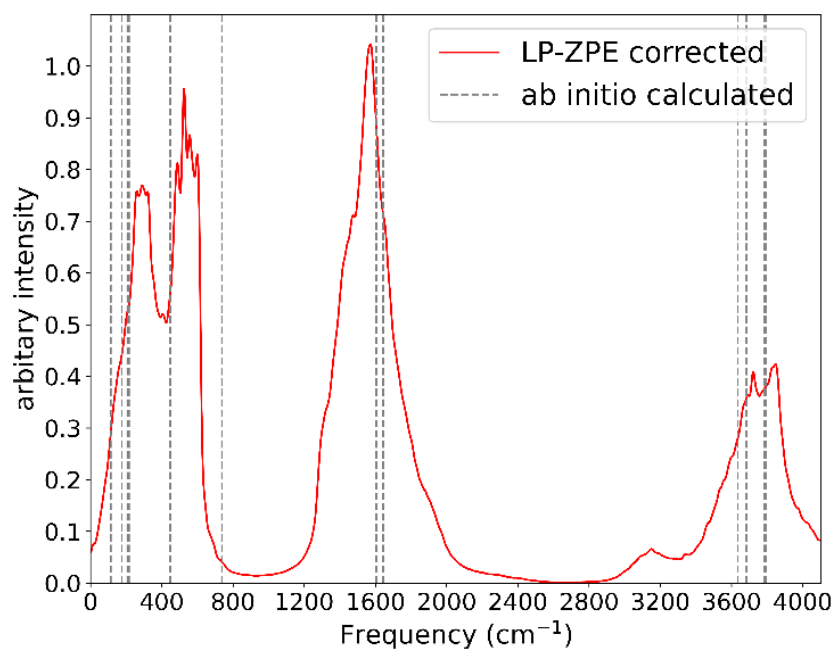

Figure 4. The power spectra are calculated using Eq. (11) and averaged over 100 classical trajectories with the LP-ZPE correction scheme. The grey dashed lines correspond to the scaled normal mode frequencies of the water dimer calculated in this present work. A scaling factor of 1.0999 is used for the low-frequency normal modes $\left(<1000 \mathrm{~cm}^{-1}\right)$ and 0.9604 for the high-frequency modes. ${ }^{43}$

\section{Conclusion and Outlook}

We have developed a new protocol for preventing ZPE leakage in classical trajectory simulations. The method, named local-pair (LP) ZPE correction, works by monitoring the ZPE of local pairs of atoms vibrating at high frequency. Whenever this local ZPE drops below a pre-defined threshold, an energy pool with contributions from all other atom pairs donates the leaked energy back to the local hot pair. The LP-ZPE method is built to have minimum interference in the dynamics. In particular, total energy, linear momentum, and angular momentum of the center of the mass are explicitly conserved. The LP-ZPE correction does not require Hessian calculations during dynamics, leading to a significant gain in longtimescale dynamics than most other ZPE corrections schemes. Moreover, it can be integrated with any BO-based molecular dynamics scheme.

The LP-ZPE method was tested on the water dimer system, which is well-known for showing artificial dissociation during ab initio BOMD due to ZPE leakage. If no correction is applied, the energy of the $\mathrm{OH}$ stretching modes fall below the corresponding ZPE, and the leaked energy is transferred unrealistically to the $\mathrm{O}_{1}-\mathrm{O}_{4}$ stretching mode causing dissociation of the dimer. The LP-ZPE method has been able to prevent the ZPE spilling of the $\mathrm{OH}$ stretching modes by pumping back the leaked energy into the corresponding modes while this 
energy is taken from the other modes of the dimer itself, keeping the system as a microcanonical ensemble.

Bowman et al. also applied the hard-wall sphere model to avoid ZPE leak in molecular dynamics on the water dimer system ${ }^{27}$ simulating 100 trajectories with a timestep of 0.0484 fs, and the correction was invoked at every $0.242 \mathrm{fs}$. We have also achieved similar results by applying our proposed method to prevent ZPE leak in classical trajectories with a much longer timestep of $0.5 \mathrm{fs}$, and the correction is invoked at every $10 \mathrm{fs}$. Moreover, it is a Hessian-free method making the simulation computationally more effective.

In the Hase-Miller approach ${ }^{8}$ of correcting ZPE leak by calculating instantaneous normal modes at each correction time step, the frequencies may appear imaginary. One needs to exclude these modes with imaginary frequencies from the ZPE constrain. In a similar context, in the present method, a BC atom pair may not have enough kinetic energy to donate, resulting in imaginary terms in Eq. (7). In that case, we ignore the particular pair and try to redistribute the energy in the other $\mathrm{BC}$ pairs. If no BC pair is ready to donate energy for constructing the pool from where the energy can be pumped to the leaked modes, we do not change the velocities at that time step, i.e., do not apply the correction and proceed further, hoping that the correction will be applied at the next correction time step $\left(t_{c}\right)$.

A Fortran 2008 written module enabling the LP-ZPE correction scheme is distributed freely with this article. This module can be interfaced readily with any trajectory propagator to prevent the ZPE leakage in classical trajectory-guided dynamics. 


\section{Appendix A. Conservation of total energy}

In the LP-ZPE correction scheme, the exact amount of energy given to an AH bond is removed from a pool of BC atom pairs. Thus, the total energy remains conserved. Although this conservation trivially follows from classical mechanics of a system of particles, here, we explicitly derive the corresponding equations.

In Eq. (5), $\delta \varepsilon$ is the amount of energy added to an $\mathrm{AH}$ bond. The new relative kinetic energy of the $\mathrm{AH}$ bond

$$
\begin{aligned}
K_{\text {rel new }}= & \frac{1}{2} \mu_{A H}\left(\mathbf{v}_{H_{\text {new }}}-\mathbf{v}_{A_{\text {new }}}\right)^{2} \\
= & \frac{1}{2} \mu_{A H}\left(\left(\mathbf{v}_{H_{\text {old }}}-\mathbf{v}_{A_{\text {old }}}\right)+\mu_{A H} \Delta_{A H}\left(\frac{1}{m_{H}}+\frac{1}{m_{A}}\right) \hat{\mathbf{u}}_{A H}\right)^{2} \\
= & \frac{1}{2} \mu_{A H}\left(\left(\mathbf{v}_{H_{\text {old }}}-\mathbf{v}_{A_{\text {old }}}\right)+\Delta_{A H} \hat{\mathbf{u}}_{A H}\right)^{2} \\
= & \frac{1}{2} \mu_{A H}\left(\left(\mathbf{v}_{H_{\text {old }}}-\mathbf{v}_{A_{\text {old }}}\right)+\left[\left(\left(v_{H_{\text {old }}}^{\|}-v_{A_{\text {old }}}^{\|}\right)^{2}+\left(\frac{2 \delta \varepsilon}{\mu_{A H}}\right)\right]^{1 / 2}-\left(v_{H_{\text {old }}}^{\|}-v_{A_{\text {old }}}^{\|}\right) \hat{\mathbf{u}}_{A H}\right)^{2}\right. \\
= & \frac{1}{2} \mu_{A H}\left(\left(\mathbf{v}_{H_{\text {old }}}-\mathbf{v}_{A_{\text {old }}}\right)-\left(v_{H_{\text {old }}}^{\|}-v_{A_{\text {old }}}^{\|}\right) \hat{\mathbf{u}}_{A H}+\left[\left(v_{H_{\text {old }}}^{\|}-v_{A_{\text {old }}}^{\|}\right)^{2}+\left(\frac{2 \delta \varepsilon}{\mu_{A H}}\right)\right]^{1 / 2} \hat{\mathbf{u}}_{A H}\right)^{2} \\
= & \frac{1}{2} \mu_{A H}\left(\left(v_{H_{\text {old }}}^{\perp}-v_{A_{\text {old }}}^{\perp}\right) \hat{\mathbf{v}}_{A H}+\left[\left(v_{H_{\text {old }}}^{\|}-v_{A_{\text {old }}}^{\|}\right)^{2}+\left(\frac{2 \delta \varepsilon}{\mu_{A H}}\right)^{1 / 2} \hat{\mathbf{u}}_{A H}\right)^{2}\left(\hat{\mathbf{v}}_{A H} \cdot \hat{\mathbf{u}}_{A H}=0\right)\right. \\
= & \left.\frac{1}{2} \mu_{A H}\left(\left(v_{H_{\text {old }}}^{\perp}-v_{A_{\text {old }}}^{\perp}\right)^{2}+\left(v_{H_{\text {old }}}^{\|}-v_{A_{\text {old }}}^{\|}\right)^{2}+\left(\frac{2 \delta \varepsilon}{\mu_{A H}}\right)\right)\right)^{2} \\
= & \frac{1}{2} \mu_{A H}\left(\left(\mathbf{v}_{H_{\text {old }}}-\mathbf{v}_{A_{\text {old }}}\right)^{2}+\left(\frac{2 \delta \varepsilon}{\mu_{A H}}\right)\right) \\
= & K_{\text {relold }}+\delta \varepsilon . \\
&
\end{aligned}
$$

The center-of-mass kinetic energy is

$$
\begin{aligned}
K_{c m_{\text {new }}} & =\frac{1}{2\left(m_{H}+m_{A}\right)}\left(m_{H} \mathbf{v}_{H_{\text {new }}}+m_{A} \mathbf{v}_{A_{\text {new }}}\right)^{2} \\
& =\frac{1}{2\left(m_{H}+m_{A}\right)}\left(m_{H}\left(\mathbf{v}_{H_{\text {old }}}+\frac{\mu_{A H}}{m_{H}} \Delta_{A H} \hat{\mathbf{u}}_{A H}\right)+m_{A}\left(\mathbf{v}_{A_{\text {old }}}-\frac{\mu_{A H}}{m_{A}} \Delta_{A H} \hat{\mathbf{u}}_{A H}\right)\right)^{2} \\
& =\frac{1}{2\left(m_{H}+m_{A}\right)}\left(m_{H} \mathbf{v}_{H_{\text {old }}}+m_{A} \mathbf{v}_{A_{\text {old }}}\right)^{2}=K_{c m_{\text {old }}} .
\end{aligned}
$$


Now, Eqs. (6-8) removes the same $\delta \varepsilon$ amount of energy from the $\mathrm{BC}$ atom pairs to conserve the total energy in the system. The contribution of each BC pair to construct the energy pool is proportional to the kinetic energy already present in the corresponding atom pair.

To show the conservation of energy explicitly, first, we consider a subsystem of H-A$\mathrm{B}$ arrangement in Figure 5. In this simplified triatomic system, the energy is added to the AH bond and removed from the $\mathrm{AB}$ atom pair. Hence atom $\mathrm{A}$ is corrected twice. Since the corrections do not change the coordinate, only velocities, it is sufficient to inspect the kinetic energies only.

Initially, the three atoms with masses $m_{H}, m_{A}, m_{B}$ have velocities $\mathbf{v}_{H_{\text {old }}} \mathbf{v}_{A_{\text {old }}}, \mathbf{v}_{B_{\text {old }}}$ and the kinetic energy of the subsystem is

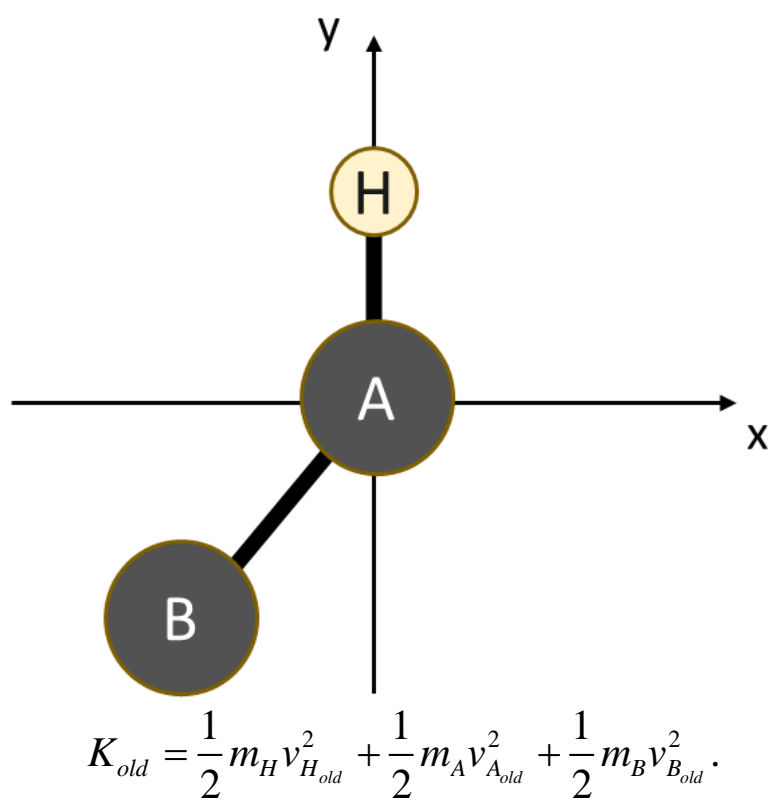

Figure 5. Atom arrangments in the subsystem H-A-B.

First, we add an amount $\delta \varepsilon$ of energy to the AH bond.

$$
\begin{aligned}
& \mathbf{v}_{H_{\text {new }}(1)}=\mathbf{v}_{H_{\text {old }}}+\frac{\mu_{A H}}{m_{H}} \Delta_{A H} \hat{\mathbf{u}}_{A H} \\
& \mathbf{v}_{A_{\text {new }}(1)}=\mathbf{v}_{A_{\text {old }}}-\frac{\mu_{A H}}{m_{A}} \Delta_{A H} \hat{\mathbf{u}}_{A H}
\end{aligned}
$$


where

$$
\Delta_{A H}=\left[\left(v_{H_{\text {old }}}^{\|}-v_{A_{\text {old }}}^{\|}\right)^{2}+\left(\frac{2 \delta \varepsilon}{\mu_{A H}}\right)\right]^{1 / 2}-\left(v_{H_{\text {old }}}^{\|}-v_{A_{\text {old }}}^{\|}\right) .
$$

For a two-body system under a central force, the kinetic energy can be written as a summation of the kinetic energy of the center-of-mass and the kinetic energy around the center-of-mass of the system.

$$
\begin{aligned}
K_{A H_{\text {old }}} & =\frac{1}{2} m_{A} v_{A_{\text {old }}}^{2}+\frac{1}{2} m_{H} v_{H_{\text {old }}}^{2} \\
& =\frac{1}{2} M_{A H}\left(\frac{m_{A} \mathbf{v}_{A_{\text {old }}}+m_{H} \mathbf{v}_{H_{\text {old }}}}{M_{A H}}\right)^{2}+\frac{1}{2} \mu_{A H}\left(\mathbf{v}_{A_{\text {old }}}-\mathbf{v}_{H_{\text {old }}}\right)^{2} \quad M_{A H}=\left(m_{A}+m_{H}\right) \\
& =K_{c m_{\text {old }}}+K_{\text {relold }} .
\end{aligned}
$$

Accordingly, the kinetic energy in the $\mathrm{AH}$ bond after correction

$$
\begin{aligned}
K_{A H_{\text {new }}(1)} & =\frac{1}{2} m_{A} v_{A_{\text {new }}(1)}^{2}+\frac{1}{2} m_{H} v_{H_{\text {new }}(1)}^{2} \\
& =\frac{1}{2} M_{A H}\left(\frac{m_{A} \mathbf{v}_{A_{\text {new }}(1)}+m_{H} \mathbf{v}_{H_{\text {new }}(1)}}{M_{A H}}\right)^{2}+\frac{1}{2} \mu_{A H}\left(\mathbf{v}_{A_{\text {new }}(1)}-\mathbf{v}_{H_{\text {new }}(1)}\right)^{2} \\
& =K_{c m_{\text {new }}(1)}+K_{\text {rel } l_{\text {new }}(1)} .
\end{aligned}
$$

Using Eqs. (13) and (14), we obtain

$$
K_{A H_{\text {new }}(1)}=K_{A H_{\text {old }}}+\delta \varepsilon
$$

Since the velocity of atom B is not changed yet, the total kinetic energy is

$$
K_{\text {new }(1)}=K_{\text {old }}+\delta \varepsilon
$$

Thereafter in the second correction step, we remove energy from the $\mathrm{AB}$ atom pair by the usual protocol:

$$
\begin{aligned}
& \mathbf{v}_{A_{\text {new }}(2)}=\mathbf{v}_{A_{\text {new }}(1)}+\frac{\mu_{A B}}{m_{A}} \Delta_{A B} \hat{\mathbf{u}}_{A B} \\
& \mathbf{v}_{B_{\text {new }}(2)}=\mathbf{v}_{B_{\text {new }}(1)}-\frac{\mu_{A B}}{m_{B}} \Delta_{A B} \hat{\mathbf{u}}_{A B}
\end{aligned}
$$

where 


$$
\Delta_{A B}=\left[\left(v_{A_{\text {new }}(1)}^{\|}-v_{B_{\text {old }}}^{\|}\right)^{2}-\left(\frac{2 \delta \varepsilon}{\mu_{A B}}\right)\right]^{1 / 2}-\left(v_{A_{\text {new }}(1)}^{\|}-v_{B_{\text {old }}}^{\|}\right)
$$

Since B atom is not corrected in the first step, $\mathbf{v}_{B_{\text {new }}(1)}=\mathbf{v}_{B_{\text {old }}}$. The energy is taken only from $\mathrm{AB}$ atom pair so that the weight factor $f_{A B}$ should be unity.

Finally, the kinetic energy after this correction is

$$
K_{\text {new }(2)}=K_{\text {new }(1)}-\delta \varepsilon=K_{\text {old }} .
$$

It is important to note that even the double correction of energy on atom A does not destroy energy conservation.

In practice, instead of a single $\mathrm{AB}$ atom pair, the energy is pumped from several $\mathrm{BC}$ atom pairs. In that case, after applying the correction to the first pair, the total kinetic energy is

$$
\begin{aligned}
K_{\text {new }(N+1)} & =K_{\text {new }(1)}-\sum_{B C} \delta \varepsilon f_{B C} \\
& =K_{\text {new }(1)}-\sum_{B C} \delta \varepsilon\left(\frac{\left(v_{B_{\text {old }}}^{\|}-v_{C_{\text {old }}}^{\|}\right)^{2}}{\sum_{B^{\prime} C^{\prime}}\left(v_{B_{\text {old }}^{\prime}}^{\|}-v_{C_{\text {old }}^{\prime}}^{\|}\right)^{2}}\right) \\
& =K_{\text {new }(1)}-\delta \varepsilon=K_{\text {old }} .
\end{aligned}
$$

\section{Appendix B. Conservation of linear and angular momentum}

The total momentum and the total angular momentum of the H-A-B subsystem (Figure 5) before the correction are

$$
\begin{gathered}
\mathbf{p}_{\text {old }}=m_{H} \mathbf{v}_{H_{\text {old }}}+m_{A} \mathbf{v}_{A_{\text {old }}}+m_{B} \mathbf{v}_{B_{\text {old }}} . \\
\mathbf{L}_{\text {old }}=\mathbf{r}_{H} \times \mathbf{p}_{H_{\text {old }}}+\mathbf{r}_{A} \times \mathbf{p}_{A_{\text {old }}}+\mathbf{r}_{B} \times \mathbf{p}_{B_{\text {old }}} .
\end{gathered}
$$

After adding energy to the AH bond, we can use Eq. (16) to verify the total momentum remains constant as: 


$$
\begin{aligned}
\mathbf{p}_{\text {new }(1)} & =m_{H} \mathbf{v}_{H_{\text {new }}(1)}+m_{A} \mathbf{v}_{A_{\text {new }}(1)}+m_{B} \mathbf{v}_{B_{\text {old }}} \\
& =m_{H} \mathbf{v}_{H_{\text {old }}}+\mu_{A H} \Delta_{A H} \hat{\mathbf{u}}_{A H}+m_{A} \mathbf{v}_{A_{\text {old }}}-\mu_{A H} \Delta_{A H} \hat{\mathbf{u}}_{A H}+m_{B} \mathbf{v}_{B_{\text {old }}} \\
& =\mathbf{p}_{\text {old }}
\end{aligned}
$$

and the angular momentum also does not change

$$
\begin{aligned}
\mathbf{L}_{\text {new }(1)} & =\mathbf{r}_{H} \times \mathbf{p}_{H_{\text {new }}(1)}+\mathbf{r}_{A} \times \mathbf{p}_{A_{\text {new }}(1)}+\mathbf{r}_{B} \times \mathbf{p}_{B, \text { old }} \\
& =\mathbf{r}_{H} \times\left(m_{H} \mathbf{v}_{H_{\text {old }}}+\mu_{A H} \Delta_{A H} \hat{\mathbf{u}}_{A H}\right)+\mathbf{r}_{A} \times\left(m_{A} \mathbf{v}_{A_{\text {old }}}-\mu_{A H} \Delta_{A H} \hat{\mathbf{u}}_{A H}\right)+\mathbf{r}_{B} \times \mathbf{p}_{B, \text { old }} \\
& =\mathbf{r}_{H} \times \mathbf{p}_{H_{\text {old }}}+\mathbf{r}_{A} \times \mathbf{p}_{A_{\text {old }}}+\mathbf{r}_{B} \times \mathbf{p}_{B_{\text {old }}}+\left(\mathbf{r}_{H}-\mathbf{r}_{A}\right) \times\left(\mu_{A H} \Delta_{A H} \hat{\mathbf{u}}_{A H}\right) \\
& =\mathbf{L}_{\text {old }}+r_{A H} \mu_{A H} \Delta_{A H} \hat{\mathbf{u}}_{A H} \times \hat{\mathbf{u}}_{A H} \\
& =\mathbf{L}_{\text {old }} .
\end{aligned}
$$

Similarly, Eq. (22) can be used to check the constancy of total momentum after correcting the $\mathrm{AB}$ atom pair as:

$$
\begin{aligned}
\mathbf{p}_{\text {new }(2)} & =m_{H} \mathbf{v}_{H, \text { new }(1)}+m_{A} \mathbf{v}_{A_{\text {new }}(2)}+m_{B} \mathbf{v}_{B_{\text {new }}(2)} \\
& =m_{H} \mathbf{v}_{H, \text { new }(1)}+m_{A} \mathbf{v}_{A, \text { new }(1)}+\mu_{A B} \Delta_{A B} \hat{\mathbf{u}}_{A B}+m_{B} \mathbf{v}_{B_{\text {new }}(1)}-\mu_{A B} \Delta_{A B} \hat{\mathbf{u}}_{A B} \\
& =\mathbf{p}_{\text {new }(1)}=\mathbf{p}_{\text {old }} .
\end{aligned}
$$

The angular momentum also remains the same after the second correction of $\mathrm{AB}$ atom pair

$$
\begin{aligned}
\mathbf{L}_{\text {new }(2)} & =\mathbf{r}_{H} \times \mathbf{p}_{H_{\text {new }}(1)}+\mathbf{r}_{A} \times \mathbf{p}_{A_{\text {new }}(2)}+\mathbf{r}_{B} \times \mathbf{p}_{B_{\text {new }}(2)} \\
& =\mathbf{r}_{H} \times \mathbf{p}_{H_{\text {new }}(1)}+\mathbf{r}_{A} \times \mathbf{p}_{A_{\text {new }}(1)}+\mathbf{r}_{B} \times \mathbf{p}_{B_{\text {new }}(1)}+\left(\mathbf{r}_{A}-\mathbf{r}_{B}\right) \times\left(\mu_{A B} \Delta_{A B} \hat{\mathbf{u}}_{A B}\right) \\
& =\mathbf{L}_{\text {new }(1)}+r_{A B} \mu_{A B} \Delta_{A B} \hat{\mathbf{u}}_{A B} \times \hat{\mathbf{u}}_{A B} \\
& =\mathbf{L}_{\text {old }} .
\end{aligned}
$$

The formulation can be trivially extended to the general case of several $\mathrm{BC}$ atom pairs, like in the case of energy

$$
\mathbf{p}_{\text {new }(N+1)}=\mathbf{p}_{\text {old }} \text {. }
$$

\section{Acknowledgments}

The authors were financially supported the European Union's Horizon 2020 research and innovation program under grant agreement No 832237 (project SubNano). They also acknowledge the Centre de Calcul Intensif d'Aix-Marseille for granting access to its highperformance computing resources. 


\section{References}

(1) Wu, S.; Marcus, R. A. Analytical Mechanics of Chemical Reactions. V. Application to the Linear Reactive H +H2 Systems. J. Chem. Phys. 1970, 53 (10), 4026-4035. https://doi.org/10.1063/1.1673874.

(2) Truhlar, D. G. Quantum Mechanics of the H+H2 Reaction: Exact Scattering Probabilities for Collinear Collisions. J. Chem. Phys. 1970, 52 (7), 3841. https://doi.org/10.1063/1.1673570.

(3) Lu, D.; Hase, W. L. Classical Mechanics of Intramolecular Vibrational Energy Flow in Benzene. IV. Models with Reduced Dimensionality. J. Chem. Phys. 1988, 89 (11), 6723-6735. https://doi.org/10.1063/1.455346.

(4) Wang, H.; Peslherbe, G. H.; Hase, W. L. Trajectory Studies of SN2 Nucleophilic Substitution. 4. Intramolecular and Unimolecular Dynamics of the $\mathrm{Cl}----\mathrm{CH} 3 \mathrm{Br}$ and ClCH3---Br- Complexes. J. Am. Chem. Soc. 1994, 116 (21), 9644-9651. https://doi.org/10.1021/ja00100a032.

(5) Guo, Y.; Thompson, D. L.; Sewell, T. D. Analysis of the Zero-point Energy Problem in Classical Trajectory Simulations. J. Chem. Phys. 1996, 104 (2), 576-582. https://doi.org/10.1063/1.470853.

(6) Ben-Nun, M.; Levine, R. D. On the Zero Point Energy in Classical Trajectory Computations. J. Chem. Phys. 1996, 105 (18), 8136-8141. https://doi.org/10.1063/1.472668.

(7) Bowman, J. M.; Gazdy, B.; Sun, Q. A Method to Constrain Vibrational Energy in Quasiclassical Trajectory Calculations. J. Chem. Phys. 1989, 91 (5), 2859-2862. https://doi.org/10.1063/1.456955.

(8) Miller, W. H.; Hase, W. L.; Darling, C. L. A Simple Model for Correcting the Zero Point Energy Problem in Classical Trajectory Simulations of Polyatomic Molecules. $J$. Chem. Phys. 1989, 91 (5), 2863-2868. https://doi.org/10.1063/1.456956.

(9) Peslherbe, G. H.; Hase, W. L. Analysis and Extension of a Model for Constraining Zero-point Energy Flow in Classical Trajectory Simulations. J. Chem. Phys. 1994, 100 (2), 1179-1189. https://doi.org/10.1063/1.466648.

(10) Lim, K. F.; McCormack, D. A. The Conservation of Quantum Zero-point Energies in Classical Trajectory Simulations. J. Chem. Phys. 1995, 102 (4), 1705-1715. https://doi.org/10.1063/1.468697.

(11) McCormack, D. A.; Lim, K. F. Zero-Point Energy Constraints in RRKM and NonRRKM Molecules. Phys. Chem. Chem. Phys. 1999, 1 (1), 1-12. 
https://doi.org/10.1039/a808024k.

(12) Bonhommeau, D.; Truhlar, D. G. Mixed Quantum/Classical Investigation of the Photodissociation of NH3( $\tilde{A})$ and a Practical Method for Maintaining Zero-Point Energy in Classical Trajectories. J. Chem. Phys. 2008, 129 (1), 014302. https://doi.org/10.1063/1.2943213.

(13) Varandas, A. J. C.; Marques, J. M. C. Method for Quasiclassical Trajectory Calculations on Potential Energy Surfaces Defined from Gradients and Hessians, and Model to Constrain the Energy in Vibrational Modes. J. Chem. Phys. 1994, 100 (3), 1908-1920. https://doi.org/10.1063/1.466544.

(14) Xie, Z.; Bowman, J. M. Zero-Point Energy Constraint in Quasi-Classical Trajectory Calculations. J. Phys. Chem. A 2006, 110 (16), 5446-5449.

https://doi.org/10.1021/jp055861e.

(15) Brieuc, F.; Bronstein, Y.; Dammak, H.; Depondt, P.; Finocchi, F.; Hayoun, M. ZeroPoint Energy Leakage in Quantum Thermal Bath Molecular Dynamics Simulations. $J$. Chem. Theory Comput. 2016, 12 (12), 5688-5697. https://doi.org/10.1021/acs.jctc.6b00684.

(16) Mangaud, E.; Huppert, S.; Plé, T.; Depondt, P.; Bonella, S.; Finocchi, F. The Fluctuation-Dissipation Theorem as a Diagnosis and Cure for Zero-Point Energy Leakage in Quantum Thermal Bath Simulations. J. Chem. Theory Comput. 2019, 15 (5), 2863-2880. https://doi.org/10.1021/acs.jctc.8b01164.

(17) Mauger, N.; Plé, T.; Lagardère, L.; Bonella, S.; Mangaud, É.; Piquemal, J.-P.; Huppert, S. Nuclear Quantum Effects in Liquid Water at Near Classical Computational Cost Using the Adaptive Quantum Thermal Bath. J. Phys. Chem. Lett. 2021, 12 (34), 82858291. https://doi.org/10.1021/acs.jpclett.1c01722.

(18) Lee, K. L. K.; Quinn, M. S.; Kolmann, S. J.; Kable, S. H.; Jordan, M. J. T. Zero-Point Energy Conservation in Classical Trajectory Simulations: Application to H2CO. J. Chem. Phys. 2018, 148 (19), 194113. https://doi.org/10.1063/1.5023508.

(19) Townsend, D.; Lahankar, S. A.; Lee, S. K.; Chambreau, S. D.; Suits, A. G.; Zhang, X.; Rheinecker, J.; Harding, L. B.; Bowman, J. M. The Roaming Atom: Straying from the Reaction Path in Formaldehyde Decomposition. Science (80-. ). 2004, 306 (5699), 1158-1161. https://doi.org/10.1126/science.1104386.

(20) Kurosaki, Y. Energy-Flow Dynamics in the Molecular Channel of Propanal Photodissociation, $\mathrm{C} 2 \mathrm{H} 5 \mathrm{CHO} \rightarrow \mathrm{C} 2 \mathrm{H} 6+\mathrm{CO}$ : Direct Ab Initio Molecular Dynamics Study. J. Phys. Chem. A 2006, 110 (39), 11230-11236. 
https://doi.org/10.1021/jp063452s.

(21) Shepler, B. C.; Braams, B. J.; Bowman, J. M. Quasiclassical Trajectory Calculations of Acetaldehyde Dissociation on a Global Potential Energy Surface Indicate Significant Non-Transition State Dynamics. J. Phys. Chem. A 2007, 111 (34), 8282-8285. https://doi.org/10.1021/jp074646q.

(22) Shepler, B. C.; Braams, B. J.; Bowman, J. M. "Roaming” Dynamics in CH3CHO Photodissociation Revealed on a Global Potential Energy Surface. J. Phys. Chem. A 2008, 112 (39), 9344-9351. https://doi.org/10.1021/jp802331t.

(23) Crespo-Otero, R.; Barbatti, M. Recent Advances and Perspectives on Nonadiabatic Mixed Quantum-Classical Dynamics. Chem. Rev. 2018, 118 (15), 7026-7068. https://doi.org/10.1021/acs.chemrev.7b00577.

(24) Mukherjee, S.; Pinheiro Jr, M.; Demoulin, B.; Barbatti, M. Simulations of Molecular Photodynamics in Long Timescales. Philos. Trans. R. Soc. A 2022. https://doi.org/10.1098/rsta-2020-0382.

(25) Koopman, E. A.; Lowe, C. P. Advantages of a Lowe-Andersen Thermostat in Molecular Dynamics Simulations. J. Chem. Phys. 2006, 124 (20), 204103. https://doi.org/10.1063/1.2198824.

(26) Huix-Rotllant, M.; Ferré, N. An Effective Procedure for Analyzing Molecular Vibrations in Terms of Local Fragment Modes. J. Chem. Theory Comput. 2016, 12 (10), 4768-4777. https://doi.org/10.1021/acs.jctc.6b00514.

(27) Czakó, G.; Kaledin, A. L.; Bowman, J. M. A Practical Method to Avoid Zero-Point Leak in Molecular Dynamics Calculations: Application to the Water Dimer. J. Chem. Phys. 2010, 132 (16), 164103. https://doi.org/10.1063/1.3417999.

(28) Odutola, J. A.; Dyke, T. R. Partially Deuterated Water Dimers: Microwave Spectra and Structure. J. Chem. Phys. 1980, 72 (9), 5062-5070. https://doi.org/10.1063/1.439795.

(29) Lane, J. R. CCSDTQ Optimized Geometry of Water Dimer. J. Chem. Theory Comput. 2013, 9 (1), 316-323. https://doi.org/10.1021/ct300832f.

(30) Klopper, W.; M. van Duijneveldt-van de Rijdt, J. G. C.; van Duijneveldt, F. B. Computational Determination of Equilibrium Geometry and Dissociation Energy of the Water Dimer. Phys. Chem. Chem. Phys. 2000, 2 (10), 2227-2234. https://doi.org/10.1039/a910312k.

(31) Curtiss, L. A.; Frurip, D. J.; Blander, M. Studies of Molecular Association in H 2 O and D 2 O Vapors by Measurement of Thermal Conductivity. J. Chem. Phys. 1979, 71 (6), 2703-2711. https://doi.org/10.1063/1.438628. 
(32) Barbatti, M.; Ruckenbauer, M.; Plasser, F.; Pittner, J.; Granucci, G.; Persico, M.; Lischka, H. Newton-X: A Surface-Hopping Program for Nonadiabatic Molecular Dynamics. Wiley Interdiscip. Rev. Comput. Mol. Sci. 2014, 4 (1), 26-33. https://doi.org/10.1002/wcms.1158.

(33) Barbatti, M.; Granucci, G.; Ruckenbauer, M.; Plasser, F.; Crespo-Otero, R.; Pittner, J.; Persico, M.; Lischka, H. NEWTON-X: A Package for Newtonian Dynamics Close to the Crossing Seam (v. 2.2). 2018.

(34) Ahlrichs, R.; Bär, M.; Häser, M.; Horn, H.; Kölmel, C. Electronic Structure Calculations on Workstation Computers: The Program System Turbomole. Chem. Phys. Lett. 1989, 162 (3), 165-169. https://doi.org/10.1016/0009-2614(89)85118-8.

TURBOMOLE GmbH. TURBOMOLE V7.3, a Development of University of Karlsruhe and Forschungszentrum Karlsruhe GmbH, 1989-2007. 2019.

(36) Brehm, M.; Kirchner, B. TRAVIS - A Free Analyzer and Visualizer for Monte Carlo and Molecular Dynamics Trajectories. J. Chem. Inf. Model. 2011, 51 (8), 2007-2023. https://doi.org/10.1021/ci200217w.

(37) Thomas, M.; Brehm, M.; Fligg, R.; Vöhringer, P.; Kirchner, B. Computing Vibrational Spectra from Ab Initio Molecular Dynamics. Phys. Chem. Chem. Phys. 2013, 15 (18), 6608. https://doi.org/10.1039/c3cp44302g.

(38) Mukhopadhyay, A.; Cole, W. T. S.; Saykally, R. J. The Water Dimer I: Experimental Characterization. Chem. Phys. Lett. 2015, 633, 13-26. https://doi.org/10.1016/j.cplett.2015.04.016.

(39) Mukhopadhyay, A.; Xantheas, S. S.; Saykally, R. J. The Water Dimer II: Theoretical Investigations. Chem. Phys. Lett. 2018, 700, 163-175. https://doi.org/10.1016/j.cplett.2018.03.057.

(40) Morokuma, K.; Pedersen, L. Molecular-Orbital Studies of Hydrogen Bonds. An Ab Initio Calculation for Dimeric H2O. J. Chem. Phys. 1968, 48 (7), 3275-3282. https://doi.org/10.1063/1.1669604.

(41) Scheiner, S. AB Initio Studies of Hydrogen Bonds: The Water Dimer Paradigm. Annu. Rev. Phys. Chem. 1994, 45 (1), 23-56. https://doi.org/10.1146/annurev.pc.45.100194.000323.

(42) Xu, X.; Goddard, W. A. Bonding Properties of the Water Dimer: A Comparative Study of Density Functional Theories. J. Phys. Chem. A 2004, 108 (12), 2305-2313. https://doi.org/10.1021/jp035869t.

(43) Sinha, P.; Boesch, S. E.; Gu, C.; Wheeler, R. A.; Wilson, A. K. Harmonic Vibrational 
Frequencies: Scaling Factors for HF, B3LYP, and MP2 Methods in Combination with Correlation Consistent Basis Sets. J. Phys. Chem. A 2004, 108 (42), 9213-9217. https://doi.org/10.1021/jp048233q.

(44) Zhou, H.; Yang, R.; Jin, X.; Zhou, M. Infrared Spectra of the OH + and H $2 \mathrm{O}+$ Cations Solvated in Solid Argon. J. Phys. Chem. A 2005, 109 (27), 6003-6007. https://doi.org/10.1021/jp0511808.

(45) Tschumper, G. S.; Leininger, M. L.; Hoffman, B. C.; Valeev, E. F.; Schaefer, H. F.; Quack, M. Anchoring the Water Dimer Potential Energy Surface with Explicitly Correlated Computations and Focal Point Analyses. J. Chem. Phys. 2002, 116 (2), 690-701. https://doi.org/10.1063/1.1408302.

(46) Fredin, L.; Nelander, B.; Ribbegård, G. Infrared Spectrum of the Water Dimer in Solid Nitrogen. I. Assignment and Force Constant Calculations. J. Chem. Phys. 1977, 66 (9), 4065-4072. https://doi.org/10.1063/1.434478.

(47) Bentwood, R. M.; Barnes, A. J.; Orville-Thomas, W. J. Studies of Intermolecular Interactions by Matrix Isolation Vibrational Spectroscopy. J. Mol. Spectrosc. 1980, 84 (2), 391-404. https://doi.org/10.1016/0022-2852(80)90031-4.

(48) Tursi, A. J.; Nixon, E. R. Matrix-Isolation Study of the Water Dimer in Solid Nitrogen. J. Chem. Phys. 1970, 52 (3), 1521-1528. https://doi.org/10.1063/1.1673163. 


\section{Supporting information}

We have sampled 500 initial conditions from correlated quantum-harmonic oscillator distribution and thereafter, selected only 100 initial conditions for ground state BOMD simulations such that the total energy of the system lies within the energy window of ZPE \pm $0.1 \mathrm{eV}$. Hence, the trajectories are initialized with the total energies close to the ZPE of the system, neither too hot nor very cold initial conditions are considered.

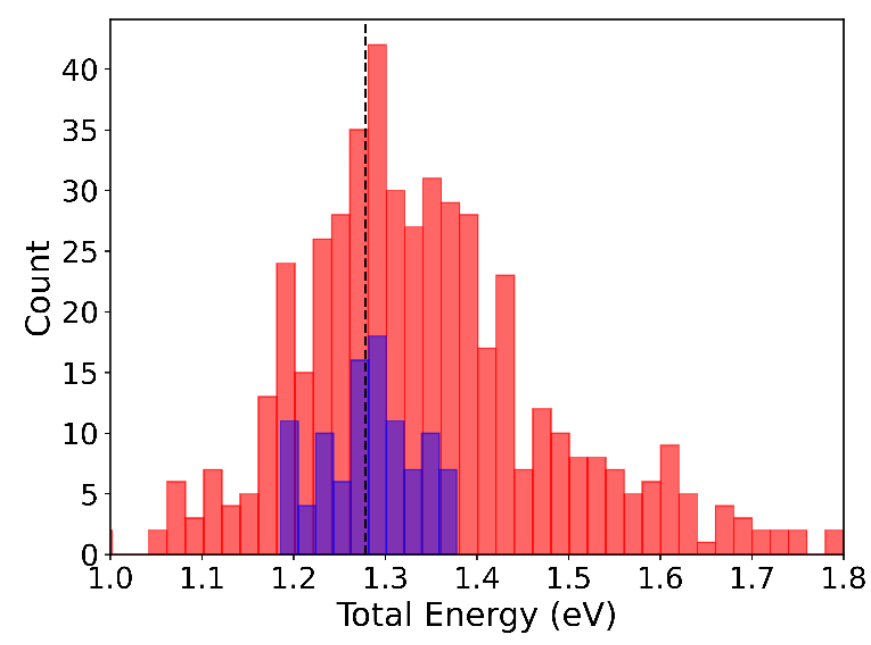

Figure S1: The total energies for 500 initial conditions sampled from the correlated quantum harmonic oscillator distribution (red histograms). The blue histograms show the energy distribution for the selected 100 initial conditions such that the total energy lies within the energy range of $\mathrm{ZPE} \pm 0.1 \mathrm{eV}$. The black dashed line marks the ZPE value of the water dimer obtained in this present calculation by MP2/aug-cc-pVDZ level of theory. 


\begin{tabular}{|c|c|c|c|}
\hline & $\begin{array}{l}\text { Present result } \\
\text { MP2/aug-cc- } \\
\text { pVDZ }\end{array}$ & $\begin{array}{l}\text { Previous theoretical } \\
\text { result }^{45} \\
\operatorname{CCSD}(\mathrm{T}) / \mathrm{TZ} 2 \mathrm{P}(\mathrm{f}, \mathrm{d}) \\
+ \text { dif }\end{array}$ & Previous expt. results ${ }^{46-48}$ \\
\hline$v_{1}$ & 139 & 131 & - \\
\hline$v_{2}$ & 155 & 154 & $(155)$ \\
\hline$v_{3}$ & 162 & 158 & - \\
\hline$v_{4}$ & 189 & 191 & $(243)$ \\
\hline$v_{5}$ & 372 & 369 & $(320)$ \\
\hline$v_{6}$ & 652 & 640 & $(520)$ \\
\hline$v_{7}$ & 1625 & 1661 & $1653(1600)$ \\
\hline$v_{8}$ & 1641 & 1686 & $1669(1618)$ \\
\hline$v_{9}$ & 3705 & 3750 & $3718(3548)$ \\
\hline$v_{10}$ & 3798 & 3827 & $3797(3626)$ \\
\hline$v_{11}$ & 3908 & 3914 & $3881(3698)$ \\
\hline$v_{12}$ & 3927 & 3934 & $3899(3714)$ \\
\hline
\end{tabular}

